\title{
Editorial
}

\section{The Japanese regional issue of Paraplegia}

It is a pleasure to welcome this the Japanese regional issue of Paraplegia.

As usual, the topics are varied and they provide a practical insight into some of the aspects of spinal disorders that are encountered in Japan.

An important nationwide epidemiological survey of spinal cord injury patients encountered in the 3 years 1990, 1991 and 1992 is provided by Shingu et al. The annual incidence was 40.2 per million of the population. One excellent outcome of the study is its value in promoting and pursuing a 'prevention campaign'.

Mizukami et al, using the Zancolli classification, provide a valuable practical account of functional activities of daily living in tetraplegic patients, in particular, in relation to locomotion and to transfers. Concerning high upper cervical spine trauma, Fujimura and associates describe anatomico-pathological lesions and neurological deficits in 82 patients who had survived the initial trauma and noted that the neurological deficits were a sign of an unstable injury and that the neurological prognosis was good.

The condition of post-traumatic syringomyelia continues to stimulate the interest of medical investigators and a new perspective, that of the flow of cerebrospinal fluid in syrinxes, is given by Tobimatsu and co-workers using magnetic resonance imaging.

To add to the literature on paraplegic wheelchair marathon racers, Dozono and colleagues provide a study on peripheral neuropathies of the upper extremities. They note the incidence of ulnar nerve lesions; and they advise competitors to wear gloves and to handle their wheelchairs properly. MRI studies are proving most invaluable in patients with a spinal cord injury and in a paper by Hayashi $e t$ al this non-invasive investigative procedure proved to be useful for cervical spinal cord injury patients where plain radiographs failed to show evidence of a fracture or a dislocation. MRI is also useful, they state, in predicting neurological recovery.

Calcification and ossification of the ligamentum flavum is not uncommon in Japan and can cause serious spinal neural disturbances. There are two relevant papers, one by Baba et al and another, a case report, by Toribatake et al.

The concluding paper, also by Baba et al, concerns a youth who did extremely well after the removal of a high cervical intradural extramedullary bronchogenic cyst.

Since writing this editorial I have been delighted to receive an editorial from $\mathrm{Dr} \mathrm{E}$ Iwatsubo, member of the editorial board of this journal, and amongst other matters it will be noted that Dr Iwatsubo refers to the dreadful catastrophe of the Kobe earthquake in Japan and provides quite alarming statistics of the casualties from that earthquake and indeed also refers to an earthquake that occurred in China in 1976. All of us concerned with Paraplegia and the International Medical Society of Paraplegia are most concerned about the Kobe tragedy and we hope that such an event will not recur. Our thoughts and best wishes go to all concerned, the injured, the relatives of those who succumbed and those who received serious injuries and to our fellow doctors and allied health workers in Japan.

We look forward to each of the regional issues of our journal and this year, in addition to the USA and Japanese issues, there will be one from France and one from Germany.

Phillip Harris Editor 